\title{
DINÁMICA DE LA HUMEDAD DEL SUELO EN UNA CUENCA AGRÍCOLA DEL SECTOR CENTRAL DE LA CUENCA DEL DUERO
}

\author{
J. MARTÍNEZ FERNÁNDEZ ${ }^{(1,3)^{*}, \text { N. SÁNCHEZ MARTÍN }}{ }^{(2,3)}$, \\ M. RODRÍGUEZ RUIZ ${ }^{(3)}$, A. SCAINI ${ }^{(3)}$ \\ ${ }^{1}$ Departamento de Geografía. Universidad de Salamanca. Cervantes, 3. 37002. Salamanca. \\ ${ }^{2}$ Departamento de Ingeniería Cartográfica y del Terreno. Universidad de Salamanca. \\ Hornos Caleros, 50. 05003. Ávila. \\ ${ }^{3}$ Centro Hispano Luso de Investigaciones Agrarias (CIALE). Universidad de Salamanca. \\ Duero, 12. 37185. Villamayor.
}

\begin{abstract}
RESUMEN. En este trabajo se estudia la dinámica de la humedad del suelo en una pequeña cuenca agrícola del sector central de la cuenca del Duero. La experiencia se llevó a cabo en la cuenca experimental de Villamor, de $1 \mathrm{~km}^{2}$, situada al SE de la provincia de Zamora. A partir de los datos medidos en once perfiles de suelo dotados de sondas TDR (Time Domain Reflectometry) a diferentes profundidades, entre los años 2002 y 2006, se analizó la evolución de la cantidad de agua edáfica y de su disponibilidad para las plantas. La comparación de los valores registrados en Villamor con los que se obtuvieron simultáneamente en la Red de Estaciones de Medición de Humedad del Suelo (REMEDHUS) en la comarca agrícola de La Guareña sobre $1300 \mathrm{~km}^{2}$, permitió valorar la representatividad de la cuenca experimental como expresión de la dinámica hidrológica de un territorio mucho más amplio del que forma parte. Los resultados del estudio llevado a cabo en la cuenca de Villamor pusieron de manifiesto los contrastes estacionales del contenido de agua en el suelo, como era de esperar bajo condiciones mediterráneas, aunque bastante más atenuados que los observados en medios próximos. La utilización del Índice de Déficit Hídrico (IDH) permitió afrontar el análisis en relación con la disponibilidad de agua para las plantas. Los datos de IDH han evidenciado unas condiciones de déficit hídrico mucho más reducido de lo que cabría esperar, dadas las condiciones semiáridas de la zona. La comparación de las series de IDH con las de otras dos cuencas con cobertura forestal y la identificación de situaciones de déficit mucho más intenso en ellas, lleva a la reflexión sobre el papel tan destacado de la cubierta vegetal en la respuesta hidrológica de determinados territorios.
\end{abstract}




\title{
Soil water dynamic in an agricultural catchment of the central sector of the Duero basin (Spain)
}

\begin{abstract}
In this work the soil moisture dynamics was estudied in a small agricultural catchment in the central sector of the Duero basin. The experience took place in the $1 \mathrm{~km}^{2}$ Villamor experimental catchment, located in the southeastern part of the Zamora province. The evolution of the soil water contenr and its availability for plants was analyzed from the data measured in eleven soil profiles equipped with TDR (Time Domain Reflectometry) probes at different depths, between 2002 and 2006. The soil moisture data recorded in Villamor which were compared against the data simultaneously registered in the Network of Soil Moisture Measurement Stations (REMEDHUS) in the agricultural region of La Guareña over $1300 \mathrm{~km}^{2}$. This comparison allowed to assess the repre-sentativeness of the experimental catchment as an expression of the hydrological dynamics of a territory larger of which is part. The results of the study carried out in Villamor have shown the seasonal water content changes in the soil, as it is expected under Mediterranean conditions. However, these changes were much more attenuated than those observed in other nearby areas. The use of the Soil Water Index (IDH) allows the analysis in terms of availability of water for the plants and enables the comparison between different areas. IDH data have shown much more reduced water deficit conditions of what might be expected, given the semi-arid conditions in the area. The comparison with series of IDH of two forested basins and the identification of deficit situations much more intense in them, leads to reflection on the very prominent role of vegetation cover in the hydrological response of certain territories.
\end{abstract}

Palabras clave: cuenca agrícola, humedad del suelo, déficit hídrico, cubierta vegetal. Key words: agricultural catchment, soil moisture, water deficit, vegetation cover.

Enviado el 22 de agosto de 2011

Aceptado el 13 de octubre de 2011

* Correspondencia: Departamento de Geografía. Universidad de Salamanca. Cervantes, 3. 37002. Salamanca.E-mail: jmf@usal.es

\section{Introducción}

El conocimiento preciso del contenido de humedad del suelo tiene un enorme interés aplicado por su implicación en procesos geomorfológicos, hidrológicos, edafológicos, ecológicos, climáticos, agronómicos, etc. Se trata, pues, de una variable crítica que juega un papel integrador entre los diferentes campos de la Geografía Física (Legates et al., 2010). Es la fuente que aporta el agua necesaria para las plantas, así como el vehículo de transporte para los nutrientes (Kirkham, 2005). Desde el punto de vista agrícola supone una inestimable herramienta de cara a gestionar tanto los cultivos que dependen de las precipitaciones como los que se basan en el regadío (Martín de Santa Olalla et al., 2005). El suelo es un medio a través del cual se produce la recarga de los acuíferos, con la fracción 
hídrica que no queda retenida por el espacio poroso. El conocimiento de la humedad edáfica antecedente permite conocer con menor incertidumbre la dinámica de generación de escorrentía ante acontecimientos de gran magnitud (Brutsaert, 2008), contribuyendo así a una mejor planificación en el ámbito de los riesgos hidrológicos. El subsistema hidro-edáfico tiene un protagonismo cada vez más destacado dentro de la modelización hidrológica. En el ámbito de la dinámica atmosférica, el estudio del agua presente en el suelo interesa especialmente debido a la influencia que ejerce en la división de la radiación incidente entre calor latente y calor sensible (Kutilek y Nielsen, 1994). Desde el punto de vista del funcionamiento de los ecosistemas, sobre todo en aquellos fuertemente limitados por el agua (Rodríguez-Iturbe y Porporato 2004), es vital la identificación del estado hídrico de los suelos y la disponibilidad del agua que almacenan. Esto es especialmente importante en relación con la gestión del medio natural en territorios como los mediterráneos, caracterizados por la escasez de recursos hídricos.

El conocimiento de la disponibilidad y dinámica del agua en los suelos es fundamental para llevar a cabo una correcta gestión de los ecosistemas naturales y de las tierras agrícolas. La determinación de la cantidad de agua disponible en cada tipo de suelo y ambiente, la identificación de valores de humedad especialmente significativos para la vegetación, la constatación de la evolución temporal de los contenidos de humedad, la identificación de los periodos críticos y el riesgo de estrés hídrico (Hernández-Santana et al., 2008), resultan de un interés fundamental para llevar a cabo una gestión adecuada de los suelos, de los ecosistemas que se desarrollan sobre ellos y para evaluar el riesgo de desertificación de un territorio (López Bermúdez, 2001).

Por todo ello, resulta de gran interés la realización de estudios sobre la dinámica de la humedad del suelo, en el mayor número de ambientes y bajo diferentes condiciones. Aspectos relacionados con la complejidad de las mediciones, el coste de los dispositivos experimentales o la dificultad de extender la información sobre esta variable más allá de los puntos de muestreo, ha hecho que, hasta hace poco, las bases de datos sobre humedad del suelo, además de tener series de corta duración, fueran escasas y se caracterizaran por un alcance espacial reducido. Afortunadamente, desde hace unos años el número de iniciativas para revertir esa situación está aumentando, y existen cada vez más experiencias de monitorización a largo plazo (Dorigo et al., 2011).

Los objetivos de este trabajo son, por un lado, estudiar la dinámica del contenido de agua del suelo en una pequeña cuenca agrícola bajo condiciones mediterráneas semiáridas en el sector central de la cuenca del Duero; por otro, analizar dicha dinámica en relación con la de otros usos de suelo y cubierta vegetal en ambientes próximos; y, por último, verificar la representatividad de la cuenca estudiada en relación con una zona agrícola más amplia.

\section{Metodología}

\subsection{Zona de estudio y dispositivos experimentales}

La cuenca experimental de Villamor (fig. 1) se localiza en el SE de la provincia de Zamora, a $35 \mathrm{~km}$ al norte de la ciudad de Salamanca $\left(41^{\circ} 14^{\prime} 25^{\prime}{ }^{\prime} \mathrm{N}-5^{\circ} 35^{\prime} 55^{\prime \prime} \mathrm{W}\right)$. La 


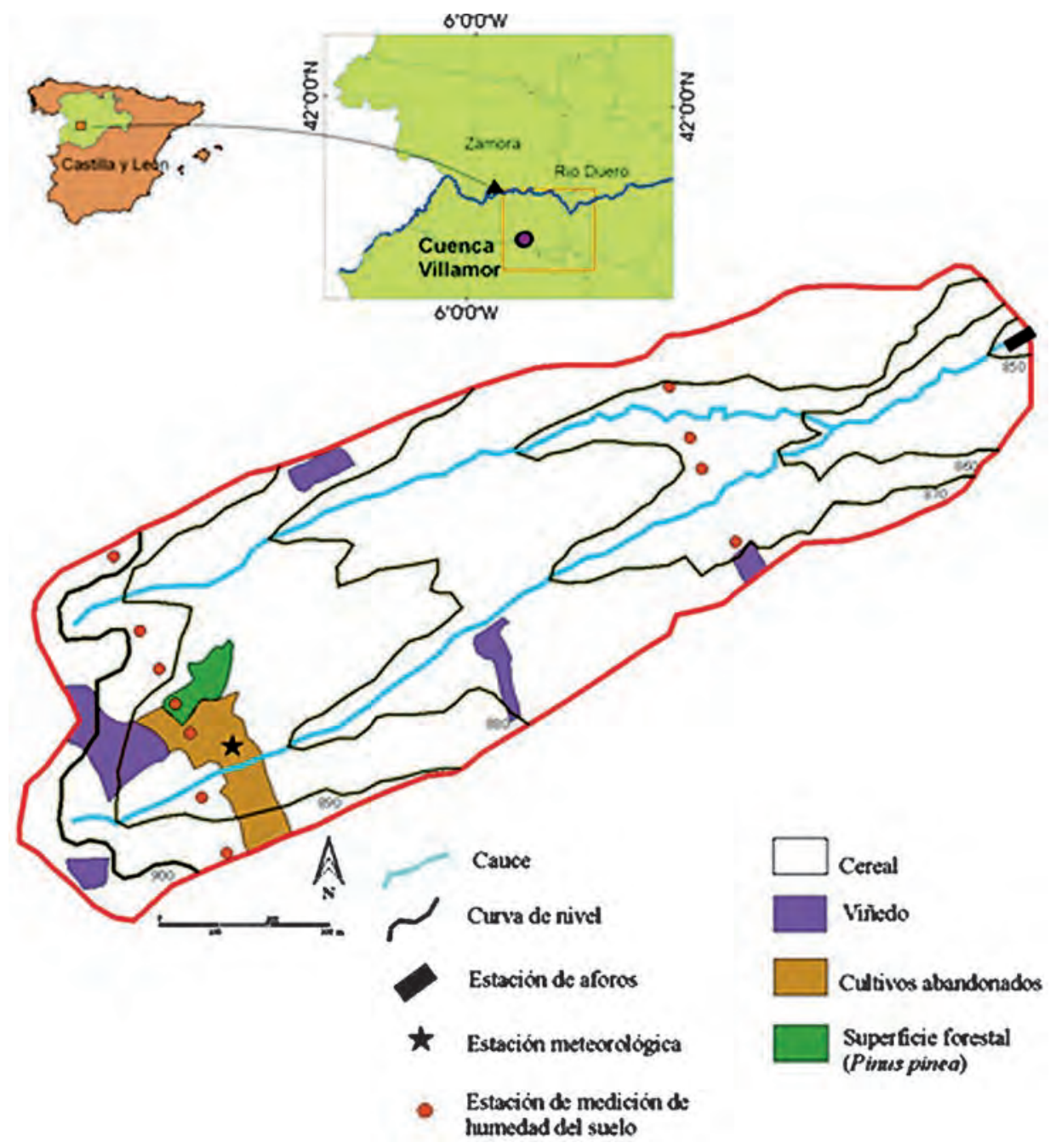

Figura 1. Localización, usos del suelo y dispositivo experimental de la cuenca experimental de Villamor (Prov. Zamora).

superficie de la cuenca es de 100 ha, y tiene una orientación suroeste-noreste y un desnivel comprendido entre los 850 y $900 \mathrm{~m}$. El sustrato geológico está compuesto por depósitos detríticos y aluviales. Luvisoles y cambisoles son los tipos de suelos más frecuentes, y presentan una textura media (tabla 1) de tipo areno-arcillosa franca, con una densidad aparente alta y un escaso contenido en materia orgánica (normalmente inferior al 1\%), como es característico de los suelos agrícolas.

El uso del suelo principal es el agrícola (Sánchez et al., 2010), con un predominio de cultivos de secano (cereal y viñedo), superficies reducidas de cultivos abandonados 
y pequeños rodales de terreno forestal con pino piñonero (Pinus pinea). El clima de este sector de la cuenca del Duero responde a la tipología de mediterráneo semiárido continentalizado. La precipitación media anual está en torno a los $400 \mathrm{~mm}$, con un coeficiente de variación anual del 20.2\%, y suelen presentarse 5 meses secos al año (marzo, junio, julio, agosto y septiembre). El mes más seco es agosto con $12 \mathrm{~mm}$ y el más húmedo diciembre con $50 \mathrm{~mm}$. La temperatura media anual es de $12.1^{\circ} \mathrm{C}$, siendo enero el mes más frío con $4^{\circ} \mathrm{C}$ y julio el más cálido con $21.1^{\circ} \mathrm{C}$. La ETP (Penman-Monteith) media anual oscila en torno a los $1000 \mathrm{~mm}$.

Tabla 1. Características de los suelos de la cuenca experimental de Villamor: textura, densidad aparente (DA) y contenido en materia orgánica (MO).

\begin{tabular}{|c|c|c|c|c|c|}
\hline Perfil/estación & $\begin{array}{c}\text { DA } \\
\left(\mathbf{g ~ c m}^{-3}\right)\end{array}$ & $\begin{array}{c}\text { Arena } \\
(\boldsymbol{\%})\end{array}$ & $\begin{array}{c}\text { Limo } \\
(\boldsymbol{\%})\end{array}$ & $\begin{array}{c}\text { Arcilla } \\
(\boldsymbol{\%})\end{array}$ & $\begin{array}{c}\text { MO } \\
(\boldsymbol{\%})\end{array}$ \\
\hline VS1 & 1.47 & 32.77 & 26.65 & 40.58 & 0.65 \\
\hline VS2 & 1.67 & 50.39 & 27.20 & 22.41 & 0.61 \\
\hline VS3 & 1.59 & 35.31 & 29.99 & 34.71 & 0.74 \\
\hline VS4 & 1.74 & 72.62 & 12.66 & 14.72 & 0.45 \\
\hline VS5 & 1.83 & 69.29 & 15.03 & 15.67 & 0.46 \\
\hline VS6 & 1.56 & 59.87 & 16.32 & 23.81 & 0.55 \\
\hline VS7 & 1.49 & 45.39 & 17.19 & 37.41 & 0.51 \\
\hline VI1 & 1.66 & 68.99 & 17.49 & 13.52 & 0.38 \\
\hline VI2 & 1.60 & 74.50 & 8.74 & 16.76 & 0.36 \\
\hline VI3 & 1.52 & 56.48 & 19.77 & 23.75 & 0.50 \\
\hline VI4 & 1.36 & 49.74 & 26.88 & 23.38 & 1.06 \\
\hline
\end{tabular}

La cuenca cuenta con una estación meteorológica automática en la que se mide temperatura y humedad del aire, velocidad y dirección del viento, radiación global y precipitación. Dispone de una estación de aforos con registro de nivel automático. La humedad del suelo se ha medido desde finales de 2001 aplicando la técnica Time Domain Reflectometry, TDR (Topp et al., 1980; Casel et al., 1994), utilizando un ecómetro modelo Tektronix $1502 \mathrm{C}$ y sondas de doble varilla calibradas en laboratorio para los suelos de la zona de estudio (Martínez Fernández y Ceballos, 2001). A finales de 2001 se instalaron en esta cuenca 11 estaciones de medición de la humedad del suelo, distribuidas en dos transectos transversales al eje mayor de la cuenca (fig. 1), uno en la parte alta y otro en la parte media. Cada estación consiste en un perfil de suelo equipado de un número variable de sondas, dependiendo de la profundidad, insertadas en posición horizontal a distintas profundidades $(5,15,25,50$ y $100 \mathrm{~cm})$. Las mediciones se realizaron a intervalos quincenales, estrategia de muestreo aceptable para dispositivos 
manuales en instalaciones experimentales a largo plazo. Los datos del presente artículo corresponden a cinco años de mediciones (2002-2006), periodo de duración poco usual y con escasos precedentes en relación con la humedad del suelo, considerado adecuado para llevar a cabo una caracterización de esta variable hidrológica (Martínez-Fernández y Ceballos, 2005). Durante esos años se han registrado unas condiciones climáticas (tabla 2) suficientemente contrastadas en relación con el contexto de la zona de estudio, habiéndose sucedido años muy húmedos (2003) y muy secos (2004).

Tabla 2. Características climáticas de los años de estudio en la cuenca de Villamor: precipitación, temperatura media anual y evapotranspiración (Penman-Monteith).

\begin{tabular}{|c|c|c|c|}
\hline Año & $\begin{array}{c}\text { Precipitación } \\
(\mathbf{m m})\end{array}$ & $\begin{array}{c}\text { Temperatura Media } \\
\text { Anual }\left({ }^{\mathbf{}} \mathbf{C}\right)\end{array}$ & $\begin{array}{c}\text { ETP } \\
(\mathbf{m m})\end{array}$ \\
\hline 2002 & 508.8 & 11.9 & 998.3 \\
\hline 2003 & 626.6 & 12.1 & 1023.9 \\
\hline 2004 & 314.1 & 11.2 & 1013.1 \\
\hline 2005 & 355.4 & 11.3 & 1106.4 \\
\hline 2006 & 522.6 & 12.0 & 1034.3 \\
\hline
\end{tabular}

El dispositivo experimental de la cuenca de Villamor se inserta en otro más amplio, que constituye REMEDHUS (Red de Medición de la Humedad del Suelo de la Universidad de Salamanca), sobre una superficie de unos $1300 \mathrm{~km}^{2}$ en la comarca de la Guareña (SE de la provincia de Zamora). Esta red experimental se compone de 20 estaciones similares a las descritas en el dispositivo de Villamor, y está en funcionamiento desde la primavera de 1999 (Martínez Fernández y Ceballos, 2003).

En la cuenca de Villamor se ha elaborado una completa base de datos sobre las propiedades físicas e hidrológicas de los suelos. Para ello, en el momento de la instalación de las sondas TDR en cada una de las estaciones de medición de la humedad y en cada punto seleccionado del perfil, se recogieron monolitos de suelo sin alterar, de $100 \mathrm{~cm}^{3} \mathrm{de}$ volumen, en los que se analizó textura, densidad aparente y contenido de materia orgánica. A partir de la determinación de la curva de retención hídrica en cada uno de esas muestras, se obtuvieron los valores correspondientes a capacidad de campo, $\theta_{\text {cc }}$ (potencial matricial $-33 \mathrm{kPa}$ ), y punto de marchitamiento, $\theta_{\mathrm{pm}}$ (potencial matricial $-1500 \mathrm{kPa}$ ).

\subsection{IDH}

Con respecto al análisis de la dinámica del agua en el suelo y en relación con los usos del suelo o la cubierta vegetal, tan interesante o más que el contenido de agua del suelo, es conocer su disponibilidad para las plantas. Por ello, se ha introducido el concepto de Índice de Déficit Hídrico (Martínez-Fernández et al., 2005), con la finalidad de 
alcanzar una mejor comprensión en el análisis de la disponibilidad de agua en el suelo y su evolución. El Índice de Déficit Hídrico (IDH) se ha definido del siguiente modo:

$$
I D H=\left(\frac{\theta-\theta_{c c}}{\theta_{d}}\right) \times 100
$$

donde $\theta$ es el contenido de humedad en un momento dado y $\theta_{\mathrm{d}}$ es el agua disponible para las plantas, es decir, la diferencia entre $\theta_{\mathrm{cc}}$, $\theta_{\mathrm{pm}}$. Cuando IDH es inferior a cero, el suelo está sometido a déficit hídrico y a partir de un valor de $-100 \%$, éste es absoluto, ya que expresa el umbral a partir del cual se sobrepasa el punto de marchitamiento, y deja de haber agua en condiciones de ser absorbida por las plantas. Valores positivos de IDH representan condiciones de exceso de agua. IDH permite obtener información de aspectos tan interesantes como son el inicio del periodo de déficit hídrico, su duración e intensidad, y si se supera en algún momento el umbral de déficit absoluto (fig. 2). Este índice permite también poder establecer comparaciones entre áreas con tipologías de suelos distintos, ya que reduce la información sobre el agua del suelo a una variable comparable a partir de unos umbrales estándar, obtenidos a partir de la curva de retención hídrica.

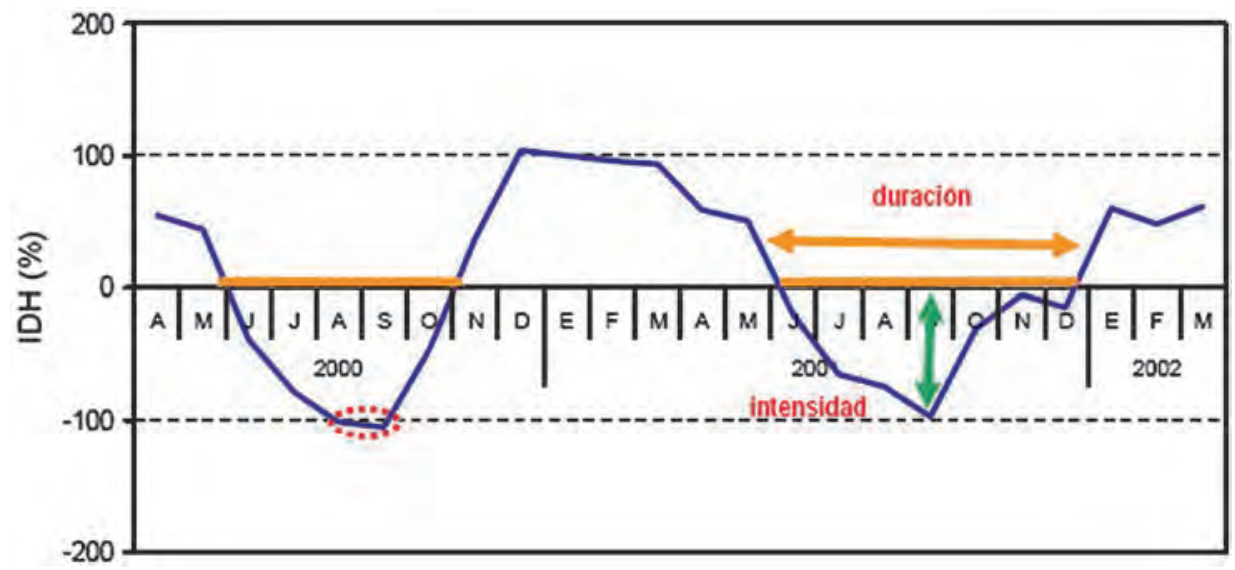

Figura 2. El Índice de Déficit Hídrico (IDH) permite obtener información sobre la duración (naranja) y la intensidad (verde) del periodo de déficit hídrico, así como de la existencia de intervalos críticos en los que se supere el umbral de déficit absoluto de disponibilidad de agua en el suelo (línea punteada en rojo).

\section{Resultados y discusión}

La evolución de la humedad media medida en los once perfiles de la cuenca de Villamor (fig. 3) muestra un gran contraste estacional, como ocurre con la mayoría de las variables hidro-climáticas en ambientes mediterráneos. Es clara y obvia la enorme dependencia de esta variable de la cantidad de precipitación, de tal manera que los valores más altos se 


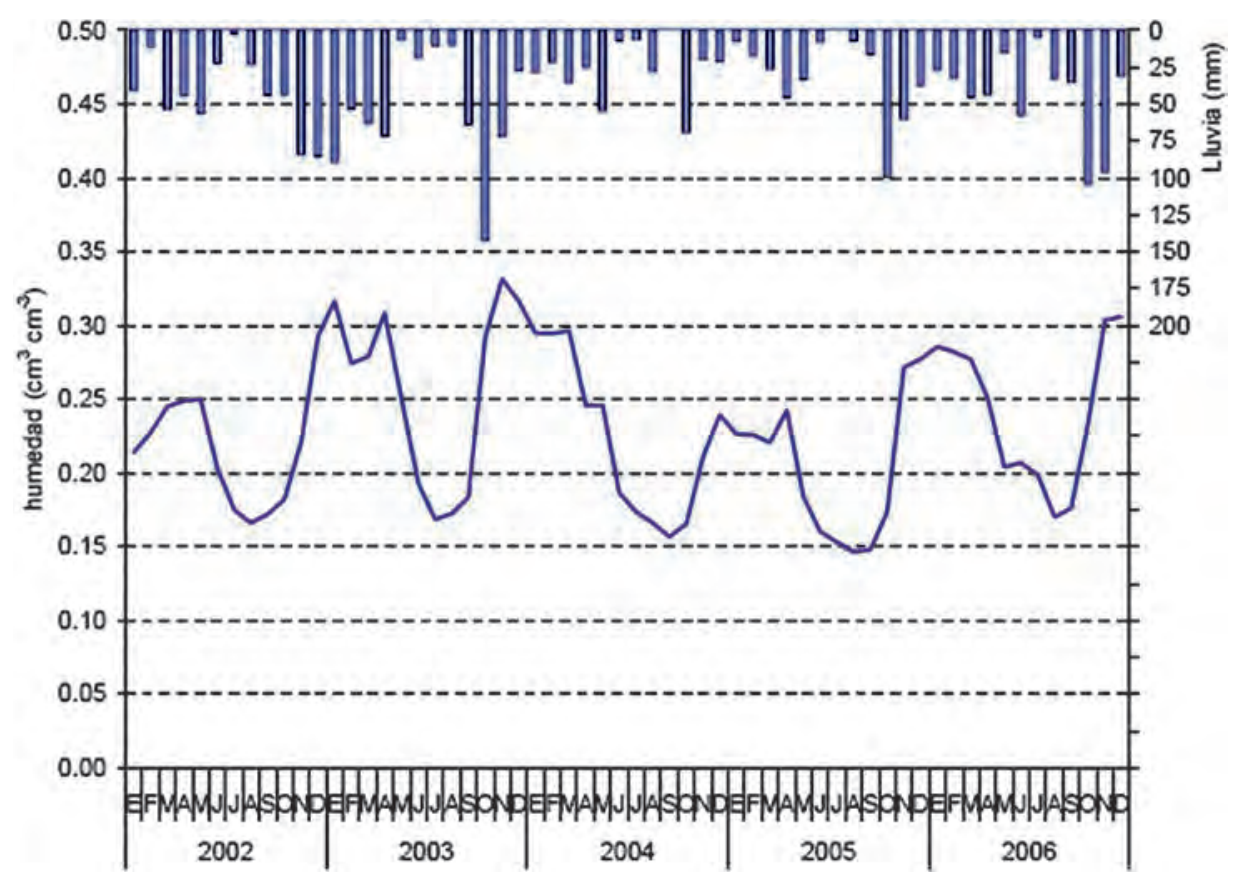

Figura 3. Evolución de la humedad del suelo media mensual y la lluvia en la cuenca experimental de Villamor entre 2002 y 2006.

registraron en 2003 y los más bajos en 2004, que son, respectivamente, los años de mayor y menor cantidad de lluvia registrada. La humedad del suelo alcanza su máximo al comienzo del invierno, entre diciembre-enero $\left(0.30-0.33 \mathrm{~cm}^{3} \mathrm{~cm}^{-3}\right)$, y el mínimo se da entre julio-agosto (alrededor de $0.15 \mathrm{~cm}^{3} \mathrm{~cm}^{-3}$ ). Sin embargo, dichos contrastes estacionales son bastante menos pronunciados que los observados en áreas próximas (Cubera y Moreno, 2007; Martínez-Fernández y Hernández-Santana, 2012), donde las condiciones son, incluso, más húmedas, dentro de un mismo contexto plenamente mediterráneo.

La explicación al comportamiento expresado en los resultados de la evolución de la cantidad de agua del suelo hay que buscarla en las características topográficas de la zona de la Guareña, las propiedades de los suelos y el uso de los mismos. Se trata de una zona llana o con pendientes muy suaves (casi siempre por debajo del 10\%), factor que condiciona severamente la circulación superficial del agua y, al mismo tiempo, favorece decisivamente la infiltración (Ceballos et al. 2002; Ceballos Barbancho et al., 2002). Los suelos son, en su mayoría, muy arenosos en superficie (hasta el $80 \%$ de arena) y en muchos de los casos tienen un horizonte B arcilloso. Esa combinación textural (areno-arcillosa franca) favorece tanto la infiltración en las capas superficiales como el almacenamiento de la humedad en el horizonte inferior. El uso del suelo predominante es el agrícola de secano ( $80 \%$ dedicado a cereales). Esto hace que, por un lado, las plantas extraigan agua exclusivamente del horizonte más superficial y que, por otro, la pérdida por absorción radicular 
sea muy débil o nula en los meses estivales por haber finalizado ya el ciclo vegetativo. Todo ello hace que, durante el verano, el contenido de agua en el perfil del suelo sea superior al que cabría esperar atendiendo a las condiciones semiáridas de la zona.

Los datos de la humedad del suelo medida en las once estaciones en la cuenca de Villamor $\left(1 \mathrm{~km}^{2}\right)$ se compararon con los valores medios de las veinte estaciones distribuidas a lo largo de la comarca agrícola de La Guareña, dentro de REMEDHUS (1300 km²), para los cinco años de observaciones. El objetivo era analizar la representatividad de una pequeña zona, seleccionada dentro de una mucho mayor, a partir de criterios de similitud topográfica, edáfica, climática y de usos del suelo. La posibilidad de encontrar áreas o puntos de muestreo representativos del valor medio de una variable difícil y costosa de medir, como es la humedad del suelo, que permitan atribuir la magnitud de dicha variable a un territorio mucho más extenso, resulta de una gran utilidad para muchas aplicaciones (Martínez-Fernández y Ceballos, 2005). Este hecho resulta especialmente provechoso cuando se trata de aplicaciones distribuidas espacialmente, como en el caso de la teledetección, pues si una localización específica demuestra representar el promedio de la región, sería factible usar esa localización en estudios posteriores (Cosh et al., 2004).

La comparación entre los datos de humedad media obtenida en la cuenca de Villamor y los registrados simultáneamente en la comarca de La Guareña, a partir de REMEDHUS, muestra un paralelismo notable (fig 4). El grado de ajuste obtenido a partir del análisis de regresión entre ambas series de datos es muy alto $\left(\mathrm{R}^{2}=0.95\right)$. Tenien-

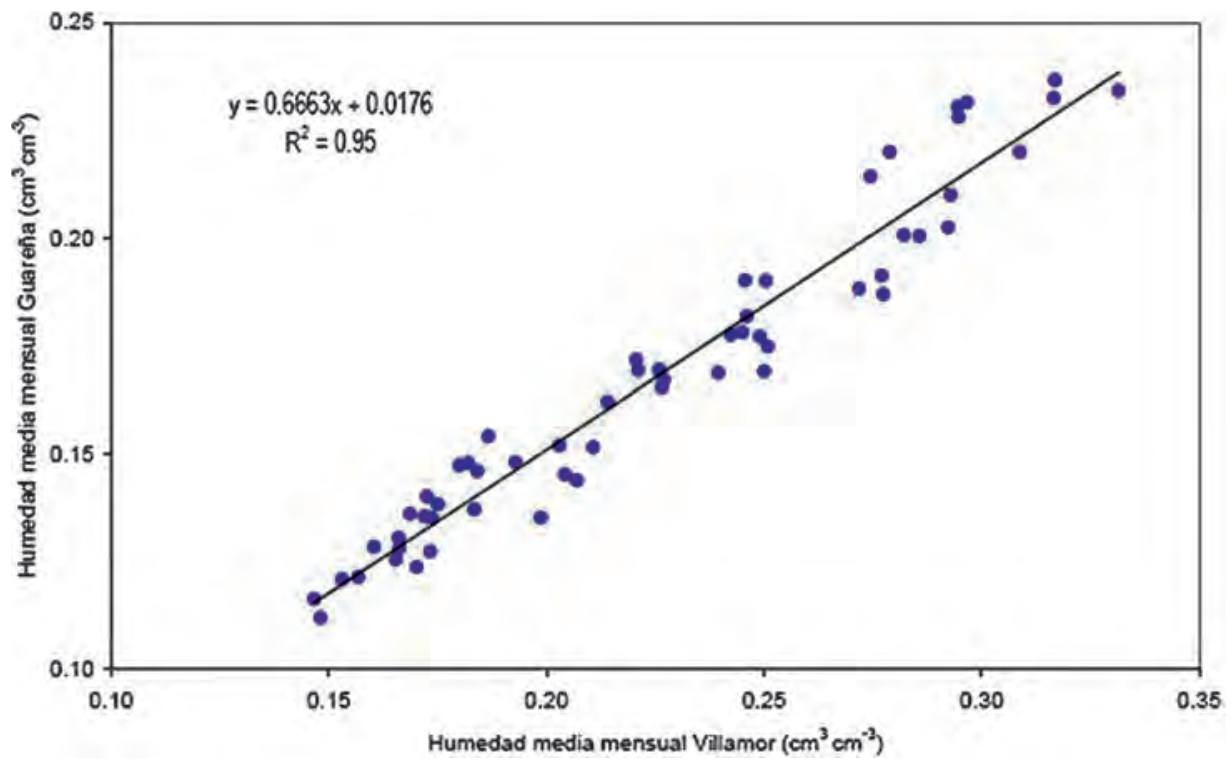

Figura 4. Relación entre la humedad media mensual medida en la cuenca experimental de Villamor y en la comarca agrícola de la Guareña, mediante la Red de Medición de la Humedad del Suelo (REMEDHUS) de la Universidad de Salamanca, entre 2002 y 2006. 
do en cuenta la diferencia de escala entre las dos zonas de muestreo $\left(1 \mathrm{~km}^{2}\right.$ frente a 1300 $\mathrm{km}^{2}$ ), resulta muy destacable la extraordinaria relación que existe entre las dos series. Esto pone en evidencia la estrecha vinculación hidrológica que existe entre esos dos ámbitos espaciales, puesta de manifiesto, en este caso, por la dinámica de la humedad del suelo. Al mismo tiempo, permite afirmar que la cuenca de Villamor es plenamente representativa, en lo que se refiere a la dinámica de la humedad del suelo, del ámbito territorial en que se inserta.

El análisis de los datos obtenidos mediante el cálculo del Índice de Déficit Hídrico (IDH) ayuda a entender mejor la significación del patrón de comportamiento de la humedad del suelo. IDH aporta una información útil en relación con diferentes procesos bio-físicos en los que el contenido de agua del suelo juega un papel destacado, como pueden ser el estrés hídrico de la vegetación o la generación de escorrentía. Durante los cinco años de observaciones (fig. 5), la duración del periodo de déficit ha oscilado entre los dos meses de 2006 y los seis de 2005, año extraordinariamente seco en la cuenca del Duero. Si bien parece que el intervalo crítico va desde junio a septiembre, hay años en los que se puede prolongar entre mayo y octubre.

Sin embargo, lo más reseñable de la evolución de IDH tiene que ver, no tanto con la duración, sino con la intensidad del déficit hídrico. El valor más bajo de IDH fue de $-49.4 \%$ y se obtuvo en agosto de 2005 . El resto de los años el mínimo ha oscilado entre $-20.6 \%$ (2006) y $-36.9 \%$ (2004). Esto significa que el déficit hídrico en los suelos de la cuenca de Villamor es muy limitado. Referido al valor medio del perfil, nunca ha llegado al umbral crítico de disponibilidad de agua para las plantas, ni ha superado siquiera la mitad de ese intervalo. Esto significa que, aun durante periodos verdaderamente extremos en términos de escasez de agua, como ocurrió en 2005, en el suelo permanece una aceptable reserva hídrica. Hay que tener en cuenta que se trató de un año especialmente significativo y que puede ilustrar muy bien la consideración de la aridez en el sector central de la cuenca del Duero, ya que entre junio y agosto apenas se recogieron $14 \mathrm{~mm}$, y en julio no llovió ni un solo día, mes en el que se estimó una ETP de 197 mm. En ese verano se registró una racha seca (sucesión de $n$ días consecutivos sin precipitación) de 47 días, una semana por encima de la media de las rachas máximas en la zona que es de 40 días (Luengo Ugidos et al., 2002).

Por tanto, en este sector central de la cuenca del Duero se observa que el periodo deficitario (IDH $<0 \%$ ) va de junio a octubre, aunque rara vez se sobrepasa el valor de $-20 \%$ (fig. 5). Bien es verdad que un análisis más pormenorizado de la zona pone en evidencia que el horizonte más superficial de los suelos puede presentar un déficit mucho más acusado. En un trabajo anterior (Martínez Fernández et al., 2007), en donde se comparaba el comportamiento de tres perfiles de suelo ubicados en la cuenca de Villamor bajo diferentes tipos de cubierta vegetal, se observó que el periodo medio de déficit $(\mathrm{IDH}<0 \%)$ a $5 \mathrm{~cm}$ de profundidad oscilaba entre los 6.9 y los 11.8 meses, bajo pinar y en suelo cultivado de cereal, respectivamente. Al mismo tiempo, se comprobó que a 50 $\mathrm{cm}$ de profundidad en el perfil, las condiciones eran muy distintas, oscilando ese periodo entre los 6.1 meses bajo los pinos y, tan solo, 0.2 meses en el suelo dedicado a cereal. En definitiva, se ha comprobado que en los suelos que se corresponden con el uso mayo- 


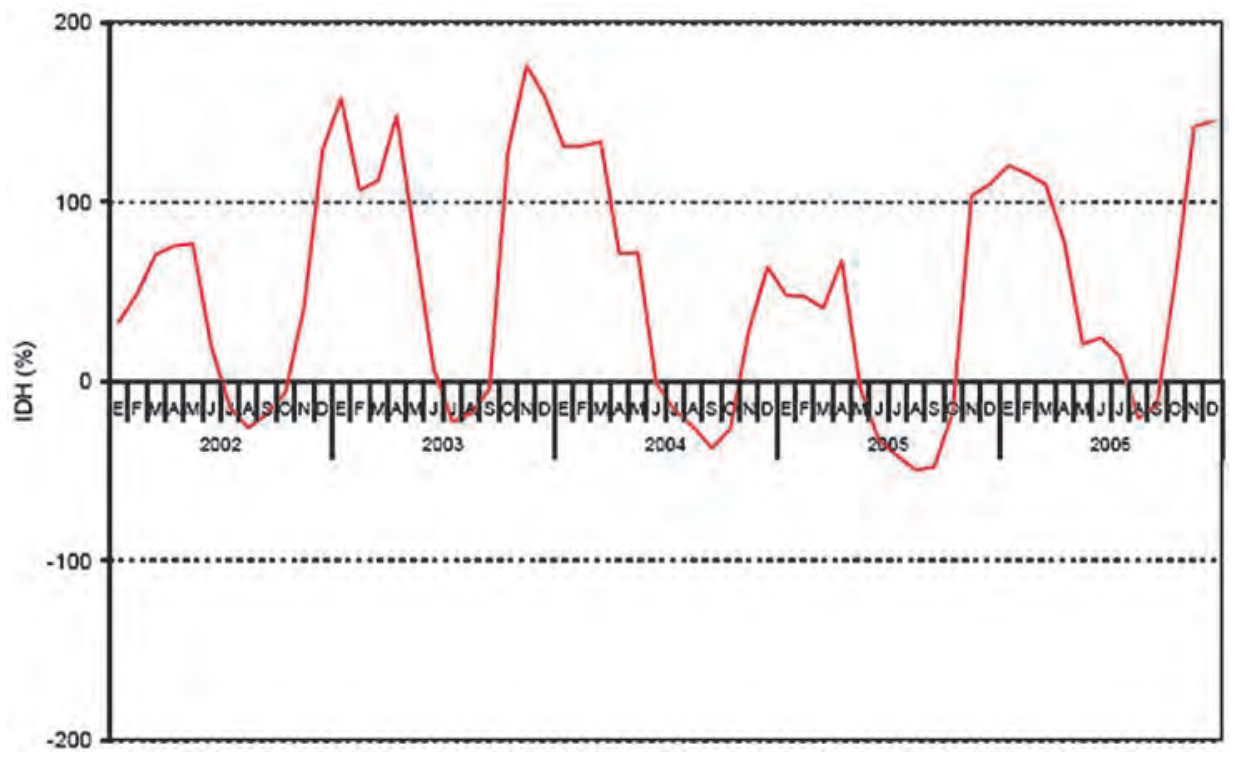

Figura 5. Evolución del Índice de Déficit Hídrico (IDH) medio mensual, en la cuenca de Villamor, entre los años 2002 y 2006.

ritario (más del 80\% del territorio se cultiva de cereal) apenas si se produce déficit hídrico, quedando restringido éste al horizonte más superficial, en relación con la distribución del sistema radicular de las gramíneas y en consonancia con su ciclo fenológico. El resto del perfil del suelo retiene una apreciable cantidad de agua durante todo el año. Esto es más reseñable, si cabe, en un contexto de aridez como el que se ha identificado en este sector de la cuenca del Duero (Luengo Ugidos et al., 2002).

Los resultados obtenidos en Villamor son todavía más reveladores si se observa lo que ocurre en ambientes próximos, siempre bajo condiciones bio-climáticas mediterráneas. En la figura 6 se compara la evolución del IDH de esta cuenca agrícola con el obtenido en otras dos cuencas forestales situadas en la provincia de Salamanca, en las que se ha medido de forma simultánea la humedad del suelo con dispositivos similares. La cuenca de Morille es una dehesa de encinas en la que se registra una lluvia media anual en torno a $500 \mathrm{~mm}$, y la de Rinconada está ocupada por un bosque denso de roble melojo (Quercus pyrenaica Willd.), en donde la precipitación media anual se aproxima a los $1000 \mathrm{~mm}$.

El análisis de disponibilidad de agua en el suelo mediante el IDH refleja la existencia de una especie de gradiente inverso al de precipitación. Si bien la duración del periodo de déficit es similar, algo que es de esperar, habida cuenta de la mediterraneidad de las tres cuencas, su alcance es muy distinto. En Villamor, donde la cantidad de precipitación es más baja, la intensidad del déficit, como ya se ha comentado, está muy atenuada. Sin embargo, en Rinconada, en el extremo lluvioso, todos los años se alcanza el valor de déficit absoluto (IDH 100\%) durante uno a tres meses. La cuenca de bosque abierto adehesa- 


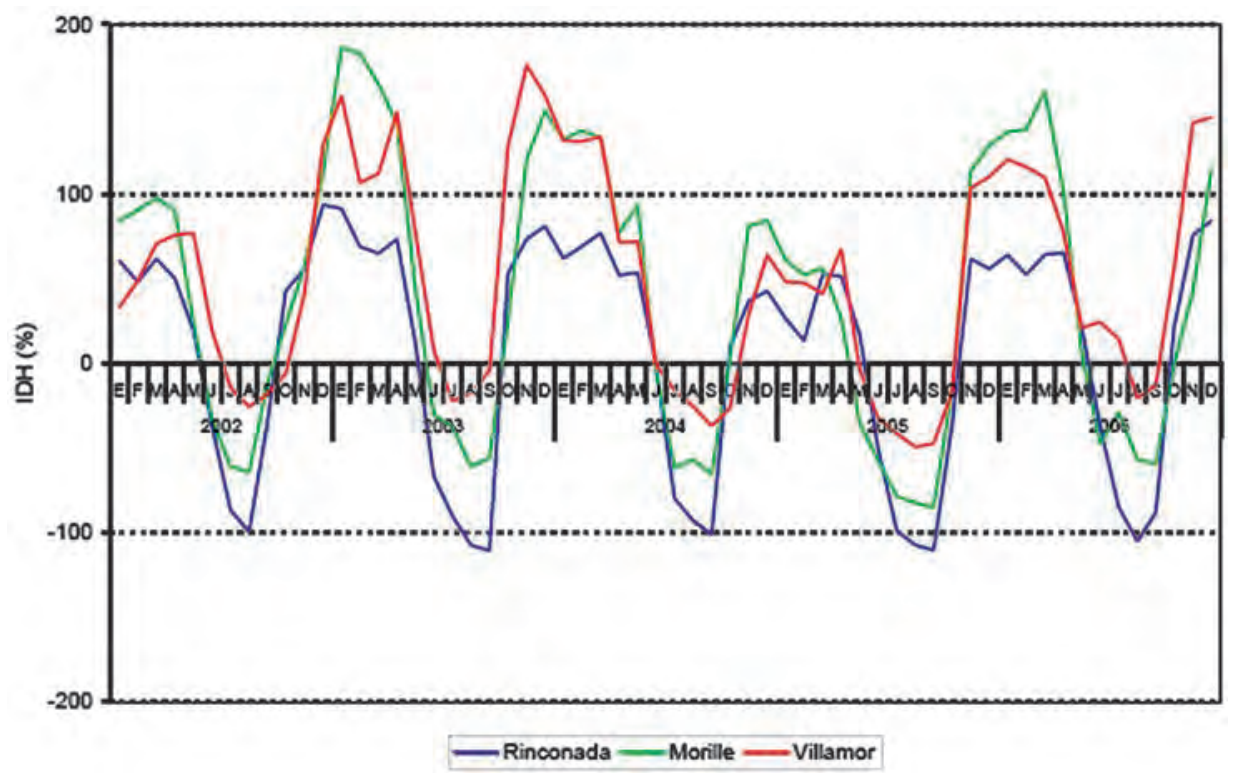

Figura 6. Evolución del Índice de Déficit Hídrico (IDH) medio mensual en las cuencas de Rinconada (bosque denso de melojo), Morille (dehesa de encinas) y Villamor (agrícola), entre los años 2002 y 2006.

do evidencia un comportamiento intermedio. Esta comparación pone de manifiesto el papel tan destacado que tiene el tipo de cubierta vegetal en el comportamiento hidrológico de un territorio (Peel, 2009). En la cuenca agrícola de Villamor, a pesar de registrarse una cantidad escasa de precipitación y una elevada evapotranspiración, se pierde poca cantidad de agua edáfica debido a que el volumen de suelo explorado por las raíces de las gramíneas se restringe al horizonte más superficial, que es al mismo tiempo el ámbito de la evaporación. Hansson y Andrín (1987) encuentran que el 85\% de la biomasa de raíces de la cebada se sitúa a menos de $30 \mathrm{~cm}$ de profundidad. En ese mismo horizonte, Manske et al. (2001) midieron el $70 \%$ del total de la longitud de raíces del trigo. Bajo condiciones ambientales mediterráneas, más del $90 \%$ de la biomasa radicular del trigo se concentra en los primeros $50 \mathrm{~cm}$ del suelo (Izzi et al., 2008). En Villamor, el resto del perfil es capaz de conservar el agua de manera adecuada, a lo que contribuyen también las características texturales del suelo, como ya se comentó anteriormente.

En las otras dos cuencas, de dedicación forestal, el tipo de vegetación predominante es capaz de explorar el perfil en su totalidad y extraer el agua de manera eficiente, sobre todo durante el periodo estival, hasta donde la disponibilidad lo permite. A diferencia de la cuenca agrícola, en donde existe una única comunidad vegetal predominante, que extrae agua exclusivamente del horizonte más superficial, en las dos cuencas forestales se dan cita un estrato arbóreo y otro herbáceo que se reparten, en el tiempo y en el espacio, su 
ámbito edáfico de exploración (Moreno et al., 2005), hasta el punto de agotar por completo cada verano el agua disponible en el primer metro del suelo (Cano Crespo, 2010).

\section{Conclusiones}

La humedad del suelo, por su condición de variable conectora de procesos, es un indicador adecuado de las condiciones hidrológicas de un territorio. En el estudio realizado en la cuenca experimental de Villamor se han puesto de manifiesto las peculiaridades del comportamiento hidrológico de una pequeña cuenca agrícola bajo condiciones mediterráneas semiáridas. El contraste climático estacional queda reflejado en la evolución del contenido de agua del suelo en los cinco años de estudio. Sin embargo, se ha podido comprobar que dicho contraste resulta bastante atenuado, si se toman en consideración las condiciones semiáridas de la zona en la que se sitúa la cuenca y, sobre todo, si se compara con lo observado en áreas relativamente próximas, también bajo condiciones mediterráneas.

A partir de la comparación de los datos medios registrados simultáneamente en Villamor y en la comarca agrícola de la que forma parte, se ha podido comprobar la representatividad hidrológica de esta micro-cuenca, en relación con el contexto en el que se sitúa. La identificación de puntos o áreas representativas es una estrategia de gran utilidad de cara a la monitorización de variables de difícil implementación.

La utilización del Índice de Déficit Hídrico ha permitido resaltar aún más las pautas de comportamiento de la humedad del suelo en la zona de estudio. Se ha observado que, a pesar de la escasez de precipitación y la elevada evapotranspiración, el suelo es capaz de conservar una cantidad notable de agua, incluso durante las épocas de mayor tensión hídrica. La comparación con los datos de dos cuencas forestales próximas, también bajo condiciones mediterráneas, ha resaltado todavía más esa peculiaridad. A la vista de esos resultados se pone de manifiesto, una vez más, la enorme relevancia hidrológica de la cubierta vegetal y del uso del suelo en determinados territorios. Ese protagonismo en relación con la dinámica de la humedad del suelo puede, como es el caso de la cuenca agrícola estudiada, superar al de los factores climáticos. Esta circunstancia es de gran interés en relación con la gestión del territorio y de los recursos hídricos, tanto de las tierras agrícolas como de aquellas que por diversos avatares ven transformada su cubierta vegetal.

\section{Agradecimientos}

Los autores quieren expresar su agradecimiento al Ministerio de Ciencia e Innovación (Proyecto AYA2010-22062-C05-02), sin cuya financiación no habría sido posible la realización de este trabajo. Al mismo tiempo expresan su reconocimiento a todas las personas (Miguel Ángel, Antonio, Pilar, Carlos Y., Vanessa, Segis, Carlos M., Virginia, Ana, Carlos P., Guido, Luciana, Pablo...) que, de un modo u otro y en un momento u otro, han colaborado para que REMEDHUS siga siendo una realidad doce años después. 


\section{Referencias bibliográficas}

Brutsaert, W. (2008). Hydrology. An introduction. Cambridge University Press. 605 pp., Cambridge.

CAno Crespo, A. (2010). Dinámica hídrica edáfica y distribución radicular en un bosque de Quercus pyrenaica Willd. del Sistema Central. Tesis de Licenciatura. Universidad de Salamanca, 142 pp., Salamanca.

Cassel, D. K., Kachanoski, R. G., Topp, G. C. (1994). Practical consideration for using TDR cable tester. Soil Technology, 7: 113-126.

Ceballos, A., Martínez-Fernández, J., Santos, F., Alonso, P. (2002). Soil-water behaviour of sandy soils under semi-arid conditions in the Duero Basin (Spain). Journal of Arid Environments, 51: 501-519.

Ceballos, A., Martínez Fernández, J., Yuste Yuste, C., Luengo Ugidos, M. A. (2002). Comportamiento hidrológico de suelos texturalmente contrastados del sector central de la cuenca del Duero. En: Aportaciones a la Geomorfología de España en el inicio del tercer milenio. (Pérez González, A., Vegas, J., Machado, M. J., Eds.) SEG. IGME, pp. 241-248. Madrid.

Cosh, M. H., Jackson, T. J., Bindlish, R., Prueger, J. H. (2004). Watershed scale temporal and spatial stability of soil moisture and its role in validating satellite estimates. Remote Sensing of Environment. 92: 427-435.

Cubera, E., Moreno, G. (2007). Effect of single Quercus ilex trees upon spatial and seasonal changes in soil water content in dehesas of central western Spain. Annals of Forest Sciences. 64: 355-364.

Dorigo, W. A., Wagner, W., Hohensinn, R., Hahn, S., Paulik, C., Drusch, M., MeCKlenburg, S., Van Oevelen, P., Robock, A., Jackson, T. (2011). The International Soil Moisture Network: a data hosting facility for global in situ soil moisture measurements. Hydrology and Earth System Sciences Discussion, 8: 1609-1663.

HANSSON, A. C., ANDRín, O. (1987). Root dynamics in barley, lucerne and meadow fescue investigated with a mini-rhizotron technique. Plant and Soil, 103: 33-38.

HernándeZ-Santana, V., David, T. S., Martínez-Fernández, J. (2008). Environmental and plant-based controls of water use in a Mediterranean oak stand. Forest Ecology and Management, 255: 3707-3715.

IzZi, G., Farahani, H. J., Bruggeman, A., Oweis, T. Y . (2008). In-season wheat root growth and soil water extraction in the Mediterranean environment of northern Syria. Agricultural Water Management, 95: 259-270.

KIRKAM, M. B. (2005). Principles of soil and plant water relationships. Elsevier Academic Press, 500 pp., London.

KutileK, M., Nielsen, D. R. (1994). Soil Hydrology. Catena-Verlag, 370 pp., Cremlingen-Destedt. 
Legates, D. R., Mahmood, R., Levia, D. F., Deliberty, T. L., Quiring, S. M., HouSER, C., Nelson, F. E. (2010). Soil moisture: A central and unifying theme in physical geography. Progress in Physical Geography, 35: 65-86.

LÓPEZ Bermúdez, F. (2001). El riesgo de desertificación. En: Agricultura y desertificación. (Martín de Santa Olalla Mañas, F., coord.). Mundi-Prensa, pp. 15-38, Madrid.

Luengo Ugidos, M. A., Ceballos Barbancho, A., Martínez Fernández, J., Yuste Yuste, C. (2002). Las rachas secas en el sector central de la Cuenca del Duero. Investigaciones Geográficas, 27: 65-82.

Manske, G. G. B., Ortiz-Monasterio, J. I., Velk, P. L. G. (2001). Techniques for measuring genetic diversity in roots. En: Application of Physiology in Wheat Breeding (Reynolds, M. P., Ortiz-Monasterio, J. I., McNab, A., eds.). CIMMYT, pp. 208218, Mexico, D.F.

Martín de Santa Olalla, F., López Fuster, P., Calera Belmonte, A. (2005). Agua y Agronomía. Mundi-Prensa, 602 pp., Madrid.

Martínez-Fernández, J., Ceballos, A. (2001). Diseño y validación de una sonda TDR para la medición de la humedad del suelo. En: Temas de Investigación de Zona no Saturada. (López, J. J. y Quemada, M., eds.). Universidad Pública de Navarra. Gobierno de Navarra, pp. 37-43, Pamplona.

Martínez-Fernández, J., Ceballos, A. (2003). Temporal stability of soil moisture in a large-field experiment in Spain. Soil Science Society of America Journal, 67: 1647-1656.

Martínez-Fernández, J., Ceballos, A. (2005). Mean soil moisture estimation using temporal stability analysis. Journal of Hydrology, 312: 28-38.

Martínez-Fernández, J., Ceballos, A., Casado, S., Morán, C., Hernández, V. (2005). Runoff and soil moisture relationships in a small forested basin in the Sistema Central ranges (Spain). En: Geomorphological Processes and Human Impacts in River Basins. (Batalla, R. J., García, C., eds.). IAHS Publ., 299: 31-36.

Martínez-Fernández, J., Cano Crespo, A., Hernández Santana, V., Morán Tejeda, C. (2007). Evolución de la humedad del suelo bajo diferentes tipos de cubierta vegetal en la cuenca del Duero. En: Estudios en la Zona no Saturada del Suelo. Vol. VIII. (Giráldez, J. V., Jiménez, F. J., eds.). Universidad de Córdoba, pp. 275-280, Córdoba.

Martínez-Fernández, J., HeRnÁndeZ-SAnTANA, V . (2012). Soil water reserve estimation and vegetation relationships in a Mediterranean subhumid forested catchment. Hydrology Research, 43: 167-178.

Moreno, G., Obrador, J. J., Cubera, E., Dupraz, C. (2005). Fine root distribution in Dehesas of Central-Western Spain. Plant and Soil, 277: 153-162.

Peel, M. C. (2009). Hydrology: catchment vegetation and runoff. Progress in Physical Geography, 33: 837-844. 
Rodríguez-Iturbe, I., Porporato, A. (2005). Ecohydrology of Water-Controlled Eco systems. Soil Moisture and Plant Dynamics. Cambridge University Press, 442 pp., New York.

Sánchez, N., Martínez-Fernández, J., Calera, A., Torres, E., Pérez-Gutiérrez, C. (2010). Combining remote sensing and in situ soil moisture data for the application and validation of a distributed water balance model (HIDROMORE). Agricultural Water Management, 98: 69-78.

TopP, G. C., Davis, J. L., AnNAN, A. P. (1980). Electromagnetic determination of soil water content. measurement in coaxial transmission lines. Water Resources Research, 16: 574-582. 